\title{
Interpretation of vector-like quark searches: heavy gluons in composite Higgs models
}

\author{
Juan Pedro Araque, ${ }^{a}$ Nuno Filipe Castro ${ }^{a, b}$ and José Santiago ${ }^{c, 1}$ \\ ${ }^{a}$ LIP, Departamento de Física, Escola de Ciências, Universidade do Minho, \\ 4710-057 Braga, Portugal \\ ${ }^{b}$ Departamento de Física e Astronomia, Faculdade de Ciências, Universidade do Porto, \\ 4169-007 Porto, Portugal \\ ${ }^{c}$ CAFPE and Departamento de Física Teórica y del Cosmos, Universidad de Granada, \\ E-18071 Granada, Spain \\ E-mail: jaraque@lip.pt, nfcastro@lip.pt, jsantiago@ugr.es
}

\begin{abstract}
Pair production of new vector-like quarks in $p p$ collisions is considered model independent as it is usually dominated by QCD production. We discuss the interpretation of vector-like quark searches in the case that QCD is not the only relevant production mechanism for the new quarks. In particular we consider the effect of a new massive color octet vector boson with sizeable decay branching ratio into the new quarks. We pay special attention to the sensitivity of the Large Hadron Collider experiments, both in run-1 and early run-2, to differences in the kinematical distributions from the different production mechanisms. We have found that even though there can be significant differences in some kinematical distributions at the parton level, the differences are washed out at the reconstruction level. Thus, the published experimental results can be reinterpreted in models with heavy gluons by simply rescaling the production cross section.
\end{abstract}

Keywords: Beyond Standard Model, Technicolor and Composite Models

ARXIV EPRINT: 1507.05628

\footnotetext{
${ }^{1}$ Corresponding author.
} 


\section{Contents}

1 Introduction 1

2 The model 3

3 Comparison of kinematical distributions and reinterpretation of limits 6

$\begin{array}{lll}3.1 & \text { Kinematical differences at parton level } & 7\end{array}$

$\begin{array}{lll}3.2 & \text { Kinematical differences after detector simulation } & 10\end{array}$

$\begin{array}{lll}3.3 & \text { Recasting VLQ searches } & 11\end{array}$

4 Limits on heavy gluons from vector-like quark searches $\quad 16$

$\begin{array}{lll}4.1 & \text { Current limits } & 16\end{array}$

$\begin{array}{lll}4.2 & \text { Early run-2 expectations } & 19\end{array}$

5 Conclusions $\quad 20$

A Technical details of the model $\quad 22$

\section{Introduction}

The discovery of the Higgs boson [1, 2] at the Large Hadron Collider (LHC) has given us, for the first time, direct experimental access to one of the least understood aspects of elementary particle interactions, the mechanism of electroweak symmetry breaking (EWSB). The absence of a dynamical mechanism that sets the scale of EWSB in the standard model (SM) makes it difficult to conceive that the SM is the whole story. It is far more likely that there is new physics beyond the SM reponsible for the dynamical realization of EWSB, and a great deal of effort from the LHC experimental collaborations is going into searching for this possible new physics. The ingenuity of model-builders has provided us with an enormous variety of new physics models, with a wealth of different phenomenological signatures, to be searched for at the LHC. The experimental collaborations have responded to this theoretical richness in a three-fold way. First, they have made a tremendous effort in trying to cover all possible experimental signatures of known models. Second, they are providing more and more details of their experimental analyses, thus making the recasting of such analyses for different models easier. Finally, they try to interpret their results in terms of the largest possible number of different models so that they are as widely applicable as possible. A good example of this are the searches for new vector-like quarks (VLQ) that have gone from being searched for in only a few final states and interpreted in terms of a single model to combining all possible final states and reporting the corresponding bounds in terms of arbitrary branching ratios (BR), see for instance [3, 4]. 
The last point mentioned above, the interpretation of experimental results in terms of a limited number of theoretical models, remains one of the main bottlenecks in the crucial exchange of information between the theoretical and experimental communities in the search for physics beyond the SM. Important efforts have been made recently, like the use of simplified models [5] or the interpretation of the experimental results in terms of effective Lagrangians. However, even this does not always capture the relevant information for some well-motivated models, either because they are too complex to be cast in the form of a reasonably simple model or because the interpretation in terms of effective operators requires too many free parameters or is complicated by the presence of new light particles in the spectrum. The recent development of Monte Carlo and fast detector simulation tools has simplified in a great manner the reinterpretation of experimental results in terms of new models. However, a serious analysis beyond an educated estimate requires a significant amount of computing power and it will never reach the complexity and precision of a fullfledged experimental analysis.

When a new physics model has experimental signatures close to the ones that are being searched for at the LHC it is worth studying in detail how the corresponding analyses can be reinterpreted in terms of the new model. There are essentially two possibilities, either the kinematical distributions relevant for the analyses are similar enough (within the experimental precision) to the ones in one of the models that are being used for the interpretation or some of the distributions are significantly different. In the former case the reinterpretation is trivial whereas in the latter it becomes much more complicated but on the other hand there is a possible handle to distinguish the new model from the ones being used for interpretation (and to increase the sensitivity of the search to the new model). The ideal case is actually somewhere in between, with identical distributions for the variables that have been used in the experimental analyses but with significant differences in extra variables that could be eventually used to design more sensitive searches. The main goal of this article is to perform such a study in a very well motivated model. In particular we consider the reinterpretation of VLQ searches in terms of a new production mechanism, namely the s-channel exchange of a massive color octet vector boson (a heavy gluon), in composite Higgs models (CHM) with partial compositeness.

CHM [6] with partial compositeness [7] predict new VLQ, called top partners, that, in the light of the Higgs mass, are expected to be relatively light [8], with masses in the $\mathrm{TeV}$ region. The mechanism of partial compositeness, that implies a linear mixing between the elementary and composite sectors, guarantees the presence of colored resonances in the spectrum and in particular, massive color octet vectors are naturally expected. If these heavy gluons have similar masses to their electroweak counterparts, they have to be in the multi- $\mathrm{TeV}$ range (due to stringent constraints from electroweak precision data on the latter [9]) and therefore it is likely that their decay into top partners, which is favored by large couplings, is kinematically open. In this case the heavy gluon will have a sizeable width and will decay mainly into the top partners, in pairs if kinematically allowed, thus significantly reducing the sensitivity of $t \bar{t}$ resonance searches to the heavy gluon [10]. ${ }^{1}$ In

\footnotetext{
${ }^{1}$ The case in which the heavy gluon decays into a SM quark and a VLQ has been studied in [11, 12].
} 
this case VLQ searches can be the most sensitive probes of the heavy gluon (see [13] for a recent study in which bounds on the heavy gluon have been computed by recasting experimental searches for pair production of new charge 5/3 VLQ and [14] for implications on recent reported excesses at the LHC.). This is therefore an example in which we have the same final state considered in several experimental searches (VLQ pair production) but with a new production mechanism. The goal of our study is to investigate if the large mass of the heavy gluon has a significant impact on the kinematical distributions used in VLQ seaches. We will see that the relevant distributions, which depending on the region of parameter space can be significantly different at the parton level, are very similar to the ones in which only QCD production is considered, once detector and reconstruction effects are taken into account. Thus, in most of the parameter space, VLQ searches can be trivially reinterpreted in terms of a rescaling of the cross section in models with heavy gluons without the need of costly computer simulations. One can simply use the published experimental limits on the VLQ production cross section as a function of the VLQ mass, that assume QCD production, and overlay the theoretical production cross section in the model with heavy gluons to compute the limits. We then use this approach to compute the current bounds on models with a heavy gluon as derived from the LHC run-1 VLQ searches and estimate the sensitivity of the early LHC run-2 data.

The rest of the article is organized as follows. We introduce the model in section 2 . We compare the relevant kinematical distributions in a model in which QCD is the only production mechanism with the ones in our model in sections 3.1 at truth parton level and 3.2 including detector and reconstruction effects. We then discuss the effect that the differences between the kinematical distributions in both models have on the actual limits extracted in section 3.3. Section 4 is dedicated to present the current limits on the model and early run-2 expectations and we conclude in section 5 . Technical details of the model are presented in appendix A.

\section{The model}

CHM with partial compositeness represent a well-motivated class of models of dynamical EWSB. They usually predict a number of relatively light VLQ with large couplings to the top (and bottom) quark, the so-called top partners, and also new vector resonances, possibly in the octet representation of the color group that couple sizeably to the top quark and its partners. They are therefore a prime candidate to study the interplay of VLQ and heavy gluon searches that we mentioned in the introduction. For the sake of concreteness we have considered a simplified version of the Minimal CHM [15], based on the $\mathrm{SO}(5) / \mathrm{SO}(4)$ coset, with a fully composite right-handed $(\mathrm{RH})$ top quark [16]. In particular we take the $\mathrm{MCH}_{5}$ model in which the SM left-handed (LH) quarks are embedded in a 5 of SO(5) and the top partners span a 4 representation of $\mathrm{SO}(4)$. Specifically they read, in the basis we 
are considering,

$$
\left(Q_{L}^{5}\right)_{I}=\frac{1}{\sqrt{2}}\left(\begin{array}{c}
\mathrm{i} b_{L} \\
b_{L} \\
\mathrm{i} t_{L} \\
-t_{L} \\
0
\end{array}\right), \quad \Psi^{i}=\frac{1}{\sqrt{2}}\left(\begin{array}{c}
\mathrm{i}\left(B-X_{5 / 3}\right) \\
B+X_{5 / 3} \\
\mathrm{i}\left(T+X_{2 / 3}\right) \\
-T+X_{2 / 3}
\end{array}\right)
$$

where $I=1, \ldots, 5$ and $i=1, \ldots, 4$, respectively. In terms of $\mathrm{SU}(2)_{L} \times \mathrm{U}(1)_{Y}$ representations, the 4 of $\mathrm{SO}(4) \Psi$ gives rise to two doublets with hypercharges $1 / 6,(T, B)$, and $7 / 6$, $\left(X_{5 / 3}, X_{2 / 3}\right)$, respectively. The latter contains an exotic state with charge $5 / 3, X_{5 / 3}$, and a charge $2 / 3$ state, $X_{2 / 3}$ whereas $T$ and $B$ have electric charge $2 / 3$ and $-1 / 3$, respectively. After EWSB, a linear combination of $T$ and $X_{2 / 3}$, that we denote $X_{2 / 3}^{\prime}$ remains degenerate with $X_{5 / 3}$. The orthogonal combination, that we call $T^{\prime}$, and $B$ are somewhat heavier with a small mass splitting. In most of the parameter space their decay BR read

$$
\begin{aligned}
B R\left(X_{5 / 3} \rightarrow t W^{+}\right) & =B R\left(B \rightarrow t W^{-}\right)=1, \\
B R\left(X_{2 / 3}^{\prime} \rightarrow t Z\right) & \approx B R\left(X_{2 / 3}^{\prime} \rightarrow t H\right) \approx \frac{1}{2}, \\
B R\left(T^{\prime} \rightarrow t Z\right) & \approx B R\left(T^{\prime} \rightarrow t H\right) \approx \frac{1}{2} .
\end{aligned}
$$

The new ingredient with respect to the $\mathrm{MCH}_{5}$ model is the presence of the heavy gluon. We introduce it following the partial compositeness mechanism [10]. The relevant part of the Lagrangian, in the elementary-composite basis, reads

$$
\begin{aligned}
\mathcal{L}= & \bar{q}_{L} \mathrm{i} \not \partial q_{L}+\bar{t}_{R} \mathrm{i} \not D t_{R}+\bar{\Psi} \mathrm{i}(\not D+\mathrm{i} \phi) \Psi-M_{\Psi} \bar{\Psi} \Psi \\
& +\left[\mathrm{i} c_{1}\left(\bar{\Psi}_{R}\right)_{i} \gamma^{\mu} d_{\mu}^{i} t_{R}+y f\left(\bar{Q}_{L}^{5}\right)^{I} U_{I i} \Psi_{R}^{i}+y c_{2} f\left(\bar{Q}_{L}^{5}\right)^{I} U_{I 5} t_{R}+\text { h.c. }\right] \\
& -\frac{1}{2} \operatorname{Tr}\left[G_{\mu \nu}^{e}\right]^{2}-\frac{1}{2} \operatorname{Tr}\left[G_{\mu \nu}^{c}\right]^{2}+\frac{1}{2} M_{c}^{2}\left(G_{\mu}^{c}-\frac{g_{e}}{g_{c}} G_{\mu}^{e}\right)^{2} .
\end{aligned}
$$

This Lagrangian is determined by three dimensionless (order one) couplings, $y$ and $c_{1,2}$, the strong couling scale $f$, the composite gluon mass $M_{c}$ and the ratio of elementary to composite gluon couplings $g_{e} / g_{c}$. The explicit expressions of the different operators in eq. (2.5) are given in appendix A. The relevant feature for us is that the elementary gluon only couples to elementary quarks, with coupling $g_{e}$, and the composite gluon only couples to the composite quarks with coupling $g_{c}$. (For simplicity we have assumed a universal coupling of the composite gluon to the composite states. This assumption does not have any relevant implication on the main conclusions of our study.) The elementary-composite gluon system can be brought to the physical basis by means of the following rotation

$$
\left(\begin{array}{c}
G_{\mu}^{e} \\
G_{\mu}^{c}
\end{array}\right)=\left(\begin{array}{cc}
\cos \theta_{3} & -\sin \theta_{3} \\
\sin \theta_{3} & \cos \theta_{3}
\end{array}\right)\left(\begin{array}{c}
g_{\mu} \\
G_{\mu}
\end{array}\right),
$$


where the ratio of couplings fixes the mixing angle $\tan \theta_{3}=\frac{g_{e}}{g_{c}}$. After this rotation we have a massless color octet, the SM gluon $g_{\mu}$, and a heavy gluon, $G_{\mu}$, with mass

$$
M_{G}=\frac{M_{c}}{\cos \theta_{3}} .
$$

The SM gluon couples universally with coupling strength

$$
g_{s}=g_{c} \sin \theta_{3}=g_{e} \cos \theta_{3},
$$

whereas the heavy gluon has couplings to elementary and composite fields given by

$$
G \bar{\psi}_{\text {elem }} \psi_{\text {elem }}:-\frac{g_{s}^{2}}{\sqrt{g_{c}^{2}-g_{s}^{2}}}, \quad G \bar{\psi}_{\text {comp }} \psi_{\text {comp }}: \sqrt{g_{c}^{2}-g_{s}^{2}} .
$$

In the following we fix the input parameters to the following values:

$$
g_{c}=3, \quad f=800 \mathrm{GeV}, \quad c_{1}=0.7, \quad c_{2}=1.7,
$$

where the value of $g_{c}$ is fixed only to test the independence of the relevant kinematical distributions of the reconstructed objects on the presence of the heavy gluon, described in detail in the next section. In order to get the bounds on $M_{G}$ from current data and the expected reach with the early LHC run-2 data we will vary $2 \leq g_{c} \leq 5$. $y$ is fixed by the top mass and its value determines the degree of compositeness of the LH top. It is most sensitive to the value of $c_{2}$ and has a milder dependence on the mass of the top parners. For the values of the input parameters chosen above it ranges from $y \approx 0.95$ for $M_{\Psi}=600 \mathrm{GeV}$ to $y \approx 0.61$ for $M_{\Psi}=1.6 \mathrm{TeV}$. This in turns corresponds to a degree of compositeness for the LH top quark

$$
s_{L}=\frac{y f}{\sqrt{y^{2} f^{2}+M_{\Psi}^{2}}},
$$

of $s_{L}=0.78$ and $s_{L}=0.29$, respectively. As it will become clear in the following sections, our goal is to show that the kinematical distributions of the reconstructed objects in VLQ searches are not affected in a significant way by the presence of the heavy gluon. This effect is not very sensitive to the degree of compositeness of the LH top quark, a fact that we implicitly check by not keeping $s_{L}$ fixed in our analyses.

The only remaining parameters are the values of the top partners and heavy gluon masses, $M_{\Psi}$ and $M_{G}$, and the behaviour of the light generations. Regarding the masses we will vary them in the relevant range

$$
600 \mathrm{GeV} \leq M_{\Psi} \leq 1.6 \mathrm{TeV}, \quad 1.5 \mathrm{TeV} \leq M_{G} \leq 4.5 \mathrm{TeV} .
$$

Finally, for simplicity we assume that the first two generations and the RH bottom quark are purely elementary.

One final comment to fully characterize our model is the fact that, due to the large number of decay channels into top partners and the large coupling to them, the width of 
the heavy gluon is typically very large, becoming quite easily comparable to $M_{G}$ itself. In that circumstance, it is compulsory to consistently include the full energy-dependent quantum width $[11,13,17]$. Due to the rapidly decaying parton distribution functions (PDF), a larger width for the heavy gluon makes it more similar to the continuum QCD production of the top partners than a narrower one. Thus, since we are trying to find how different the VLQ pair production via the heavy gluon is from the QCD one, we have considered a modification of the model in which the couplings to the composite fermions, including the RH top and the LH top and bottom, are rescaled down, when necessary, to fix the maximum width of the heavy gluon to $20 \%$ of its mass. We emphasize again that this variation goes in the direction of making the eventual differences between the two production mechanisms larger and therefore if no significant differences are found in this case, they are not expected to arise in the usual case with a wider heavy gluon. ${ }^{2}$

\section{Comparison of kinematical distributions and reinterpretation of limits}

In this section we want to investigate whether the presence of a heavy gluon in CHM affects VLQ pair production in a significant way. If it does, detailed simulations are needed to recast the result of current VLQ searches for the model at hand, although then new experimental searches might be designed to gain further sensitivity to these models. If it does not, the bounds can be trivially reinterpreted in CHM with heavy gluons by simply rescaling the corresponding cross section. In the case of a heavy gluon, one could expect that its large mass provides the VLQ with a larger energy and therefore modify the kinematical distributions of the particles they decay into. Clearly, how large the effect is depends on the relation between the heavy gluon and the VLQ masses and also on the available energy.

In order to test all these features in detail we will concentrate on one particular analysis, the VLQ pair-production searches in the $Z t$ channel [20] and display the kinematical distributions assuming production only of $X_{2 / 3}^{\prime}$. In section 3.1 we will show the differences on the main kinematical distributions at the parton level. For a better comparison we will display the distributions both normalized to their corresponding cross section and also to unit area. This latter normalization gives us a more clear picture of whether the differences can be interpreted as purely cross section rescaling or they present significant shape differences. We will then compare the distributions after hadronization, showering and detector simulation is included in section 3.2. Finally, in section 3.3 we compare the current limits from run-1 data with a full recasting of the analysis in the model versus a simple rescaling of the corresponding cross sections.

We have generated a UFO model [21] using FeynRules [22]. The simulations at parton level have been done with MG5 [23] while for hadronization/showering and detector simulation we have used pythia 6 [24] and Delphes 3 [25], respectively. We have used the default ATLAS Delphes card with the following modified parameters: the Fast Jet [26] ParameterR and the $\Delta R$ in the $b$-jets have been set to 0.4 ; the $b$-tagging efficiency has

\footnotetext{
${ }^{2}$ With this modification, the heavy gluon has a behaviour more similar to the narrower electroweak vector boson resonances, recently studied in [18]. These resonances might be responsible for the reported diboson excess if the decay into top partners is not open [19].
} 


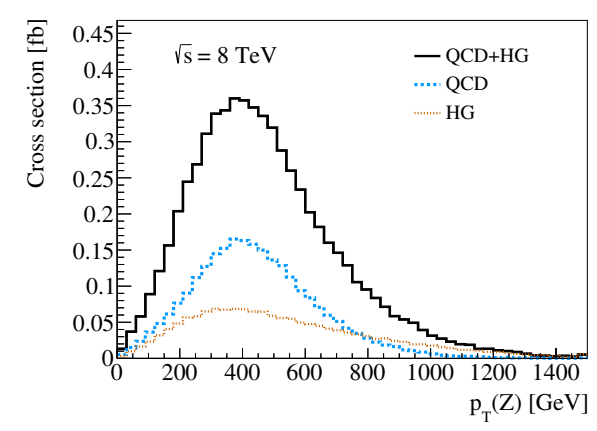

(a)

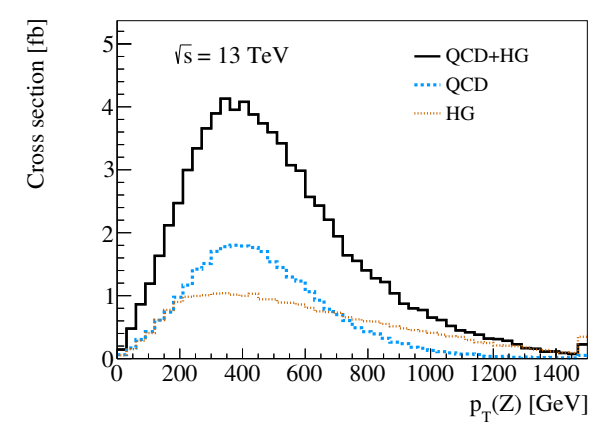

(c)

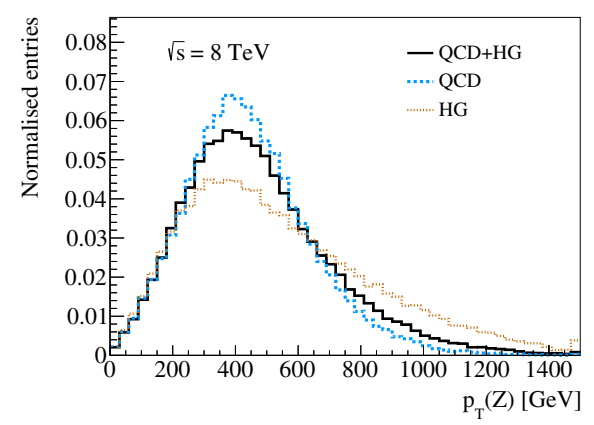

(b)

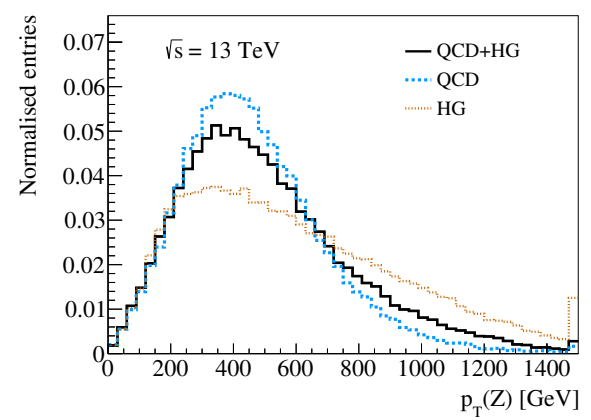

(d)

Figure 1. Distribution of the $Z$ boson transverse momentum for $\sqrt{s}=8 \mathrm{TeV}$ (top) and $13 \mathrm{TeV}$ (bottom) at parton level with $M_{G}=3.5 \mathrm{TeV}$ and $M_{X_{2 / 3}^{\prime}}=1 \mathrm{TeV}$. The plots on the left are normalized to the corresponding cross sections whereas the ones on the right are normalized to unit area.

been set to $70 \%$ and the mistag probabilities for $c$-jets and light-quark/gluon jets to $20 \%$ and $0.7 \%$, respectively.

\subsection{Kinematical differences at parton level}

In this section we explore the kinematical differences, at the parton level, between the production of a pair of vector-like quarks via $\mathrm{QCD}$ and considering the presence of a heavy gluon in the s-channel. In order to disentangle the origin of any possible kinematical differences, we compare the pair production of $X_{2 / 3}^{\prime}$ quarks via QCD (QCD), via the heavy gluon only (HG) and via both QCD and heavy gluon, including interference effects (QCD+HG). We have studied a large number of kinematical distributions for different values of the $G$ and $X_{2 / 3}^{\prime}$ masses, both at $\sqrt{s}=8$ and $13 \mathrm{TeV}$ and we will report on the most relevant ones. When showing kinematical distributions in this section, we will show the distributions normalized to their corresponding cross section on the left and the same distributions normalized to unit area on the right. The former give us an idea of the size of the different contributions whereas the latter give us intuition on how the efficiencies might change depending on the production mechanism.

In experimental searches it is common to start looking at distributions of objects in the final state that are easy to reconstruct. An example would be the $p_{\mathrm{T}}$ of the $Z$ boson in the $Z$ decay of the $X_{2 / 3}^{\prime}\left(X_{2 / 3}^{\prime} \rightarrow Z t \rightarrow Z W b\right)$. We show the $p_{\mathrm{T}}(Z)$ distribution in 


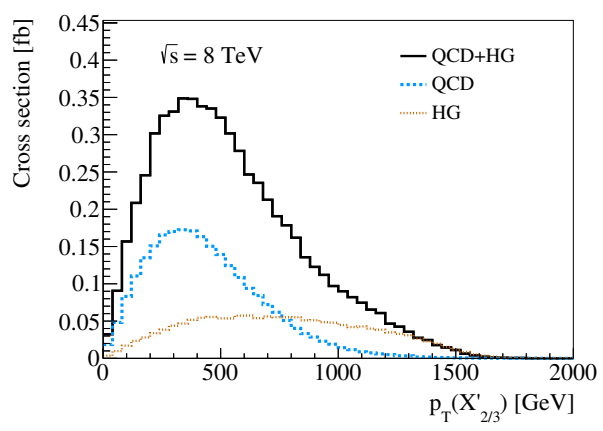

(a)

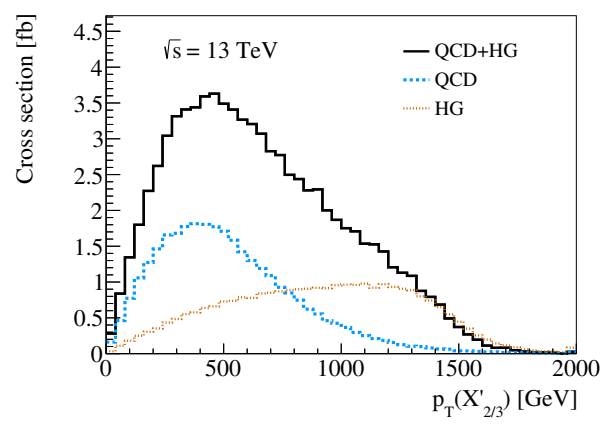

(c)

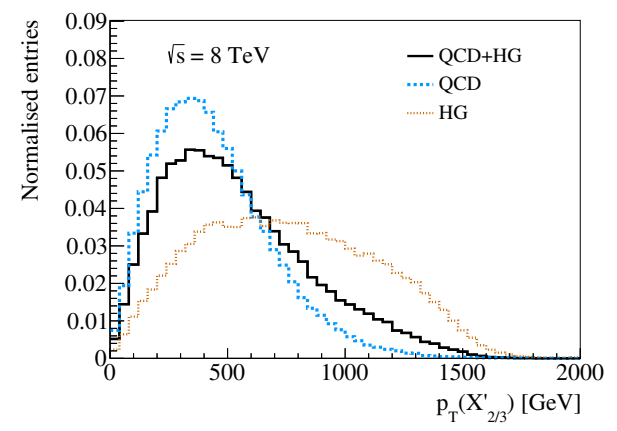

(b)

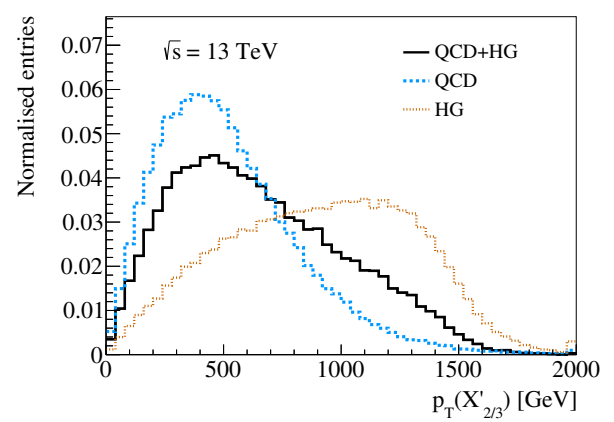

(d)

Figure 2. $X_{2 / 3}^{\prime} p_{\mathrm{T}}$ distribution for $\sqrt{s}=8$ (top) and $13 \mathrm{TeV}$ (bottom) at parton level with $M_{G}=3.5 \mathrm{TeV}$ and $M_{X_{2 / 3}^{\prime}}=1 \mathrm{TeV}$. The plots on the left column are normalized to the corresponding cross section whereas the ones on the right are normalized to unit area.

figure 1 for $\sqrt{s}=8$ and $13 \mathrm{TeV}$ at the top and bottom rows, respectively. As mentioned above, the left plots are normalized to the corresponding cross section whereas the right ones are normalized to unit area. We have fixed $M_{G}=3.5 \mathrm{TeV}$ and $M_{X_{2 / 3}^{\prime}}=1 \mathrm{TeV}$ to have a sizeable contribution from the HG channel. It can be seen that, even though there are clear differences between QCD and HG, the shapes of QCD and QCD+HG are very similar, both at 8 and $13 \mathrm{TeV}$. We have observed a similar behaviour in properties of objects near the detector such as the $p_{\mathrm{T}}$ of leptons or other vector bosons present in the decay chain.

As one could expect, kinematical distributions of objects further up the decay chain are more sensitive to the presence of the heavy gluon. We show in figures 2 and 3 the distribution of $p_{\mathrm{T}}\left(X_{2 / 3}^{\prime}\right)$ and $M\left(X_{2 / 3}^{\prime} X_{2 / 3}^{\prime}\right)$, respectively. The same layout is used with data at 8 (13) $\mathrm{TeV}$ in the top (bottom) row. The left panels normalized to the corresponding cross sections whereas the right panels are normalized to unit area and the same values of the $G$ and $X_{2 / 3}^{\prime}$ masses are chosen. As we see in the figures, the transverse momentum of the VLQ already shows some differences in shape between QCD and QCD+HG, differences that, as expected, are even larger for the invariant mass of the VLQ pair. The differences are also larger at $13 \mathrm{TeV}$ than at $8 \mathrm{TeV}$ as can be easily understood from figure 3 . Due to the PDF suppression, a heavy gluon with $M_{G}=3.5 \mathrm{TeV}$ is largely produced off-shell for $\sqrt{s}=8 \mathrm{TeV}$. At $13 \mathrm{TeV}$, on the other hand, the heavy gluon is mostly produced on-shell, with a distinctive peak in the invariant mass distribution. The kinematical distributions 


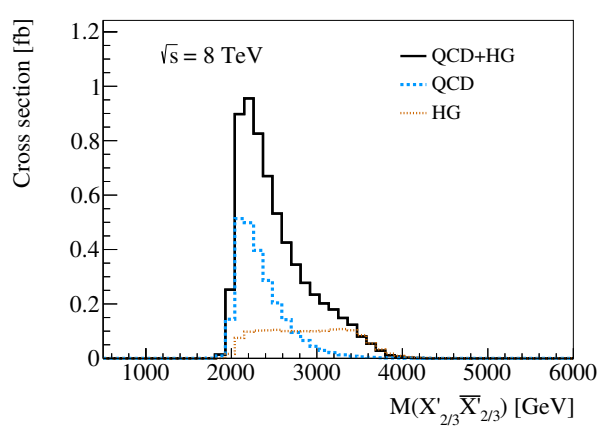

(a)

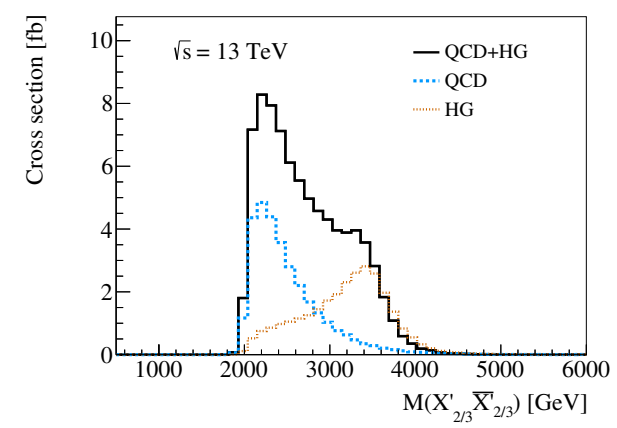

(c)

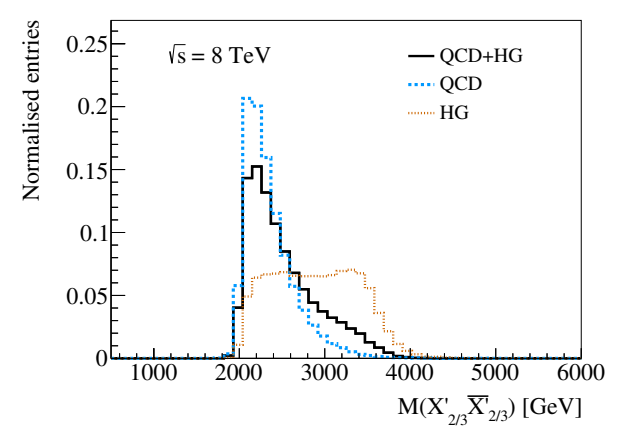

(b)

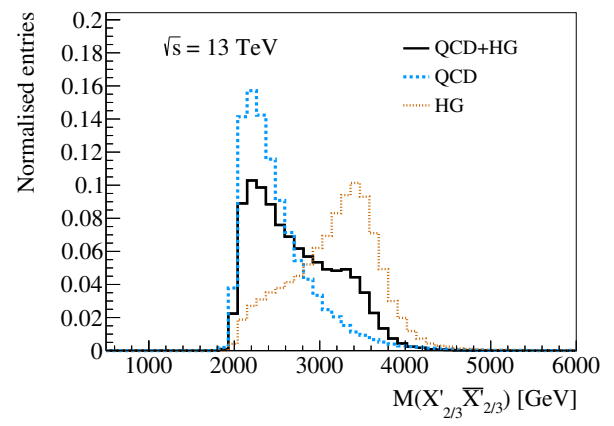

(d)

Figure 3. $X_{2 / 3}^{\prime} \bar{X}_{2 / 3}^{\prime}$ invariant mass distribution for $\sqrt{s}=8$ (top) and $13 \mathrm{TeV}$ (bottom) at parton level with $M_{G}=3.5 \mathrm{TeV}$ and $M_{X_{2 / 3}^{\prime}}=1 \mathrm{TeV}$. The plots on the left column are normalized to the corresponding cross section whereas the ones on the right are normalized to unit area.

of its decay products are therefore harder and easier to distinguish from the QCD case. Of course, there is a balance between distributions that are more easily measured but less sensitive and those that are more sensitive to the presence of the heavy gluon but more difficult to measure. Furthermore, the latter will naturally suffer a larger degradation when more realistic effects, like hadronization and detector simulation are taken into account, as discussed in section 3.2.

Before considering detector simulation we would like to point out that the presence of a heavy gluon does not always imply a harder spectrum than in QCD production. In the plots above, we chose the $M_{G}=3.5 \mathrm{TeV}$ and $M_{X_{2 / 3}^{\prime}}=1 \mathrm{TeV}$ to have a comparable contribution from QCD and HG productions with a VLQ mass compatible with current experimental bounds. In this example the heavy gluon mass is large enough to allow the VLQ to always be produced on-shell. An interesting situation occurs when the heavy gluon mass is slightly below the kinematical threshold for the VLQ pair production and the HG production dominates over the QCD one. In this case, there is still a sizeable production of VLQ pairs in which at least one of the two VLQ is slightly off-shell, thus making the corresponding spectrum softer than the one in QCD production. An example of this can be seen in figure 4 in which we show the $p_{\mathrm{T}}\left(X_{2 / 3}^{\prime}\right)$ (top) and $M_{X_{2 / 3}^{\prime} \bar{X}_{2 / 3}^{\prime}}$ (bottom) distributions for $M_{G}=2.5 \mathrm{TeV}$ and $M_{X_{2 / 3}^{\prime}}=1.3 \mathrm{TeV}$ at $\sqrt{s}=8 \mathrm{TeV}$. As usual the left plots are normalized to their corresponding cross sections and the right ones to unit area. We see 


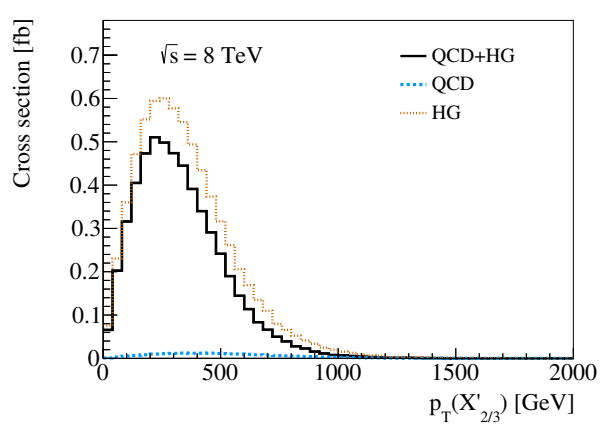

(a)

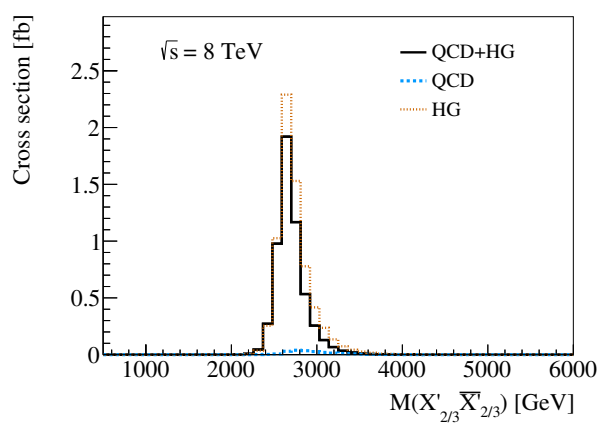

(c)

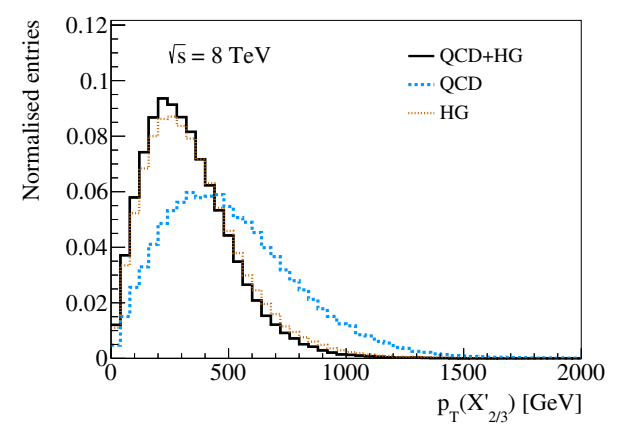

(b)

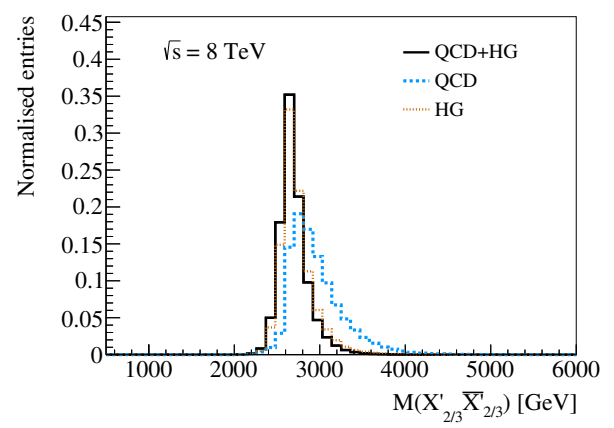

(d)

Figure 4. $X_{2 / 3}^{\prime}$ transverse momentum (top) and $X_{2 / 3}^{\prime} \bar{X}^{\prime}{ }_{2 / 3}$ invariant mass (bottom) distributions for $\sqrt{s}=8 \mathrm{TeV}$ with $M_{G}=2.5 \mathrm{TeV}$ and $M_{X_{2 / 3}^{\prime}}=1.3 \mathrm{TeV}$. The left plots are normalized to the corresponding cross sections whereas the right ones are normalized to unit area.

in the figure that the heavy gluon resonance slightly below the pair production threshold makes the spectrum softer. As a result of this the corresponding analysis efficiencies are smaller for the QCD+HG production than the ones for QCD. This effect continues at $\sqrt{s}=13 \mathrm{TeV}$ despite the larger available phase space.

\subsection{Kinematical differences after detector simulation}

Once we have explored the sensitivity of different kinematical distributions to the presence of a heavy gluon at the parton level, we consider now a more realistic set-up in which showering/hadronization and detector simulation are included. In order to produce the kinematical distributions after detector simulation the physics objects need to be properly reconstructed. We will describe our reconstruction method in detail in the next section and report here simply the corresponding kinematical distributions. Our goal is to show how the distributions used as example in the previous section get modified when detector simulation is included. However, aiming at the recostruction of the full $X_{2 / 3}^{\prime} \bar{X}_{2 / 3}^{\prime}$ system is too ambitious if one is interested in run-1 or early run-2 data. Thus, instead of the $X_{2 / 3}^{\prime} \bar{X}_{2 / 3}^{\prime}$ invariant mass distribution, we will show another distribution that naturally appears at the detector level, namely the scalar sum of the transverse momenta of all jets in the event, $H_{\mathrm{T}}$. We show in figure 5 the invariant mass of the objects that reconstruct the VLQ (top left), to show that our reconstruction method indeed captures the correct 


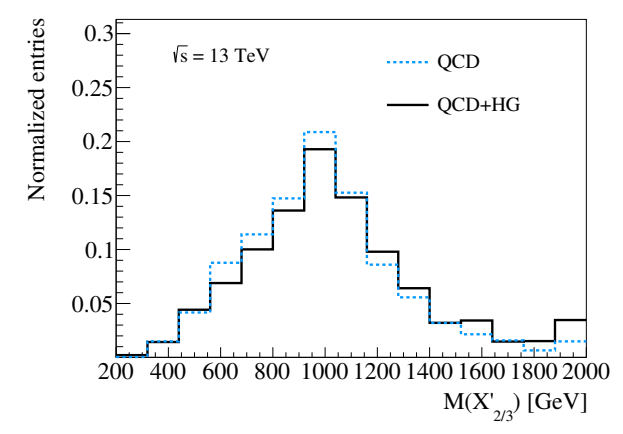

(a)

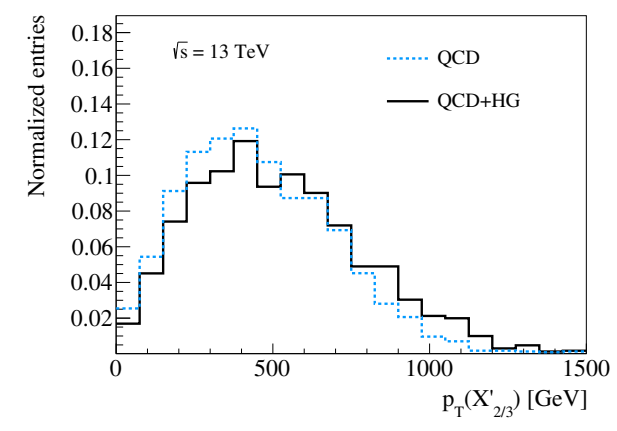

(c)

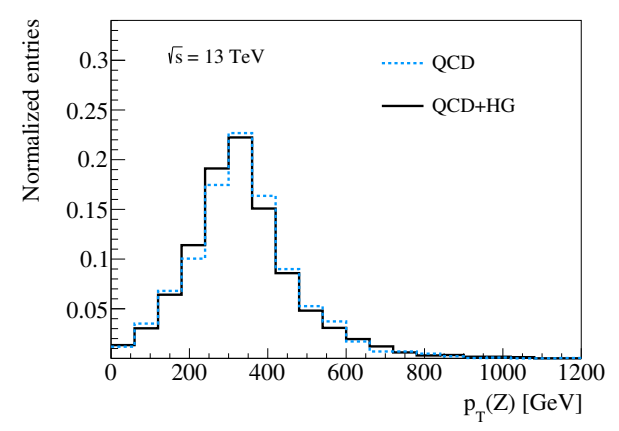

(b)

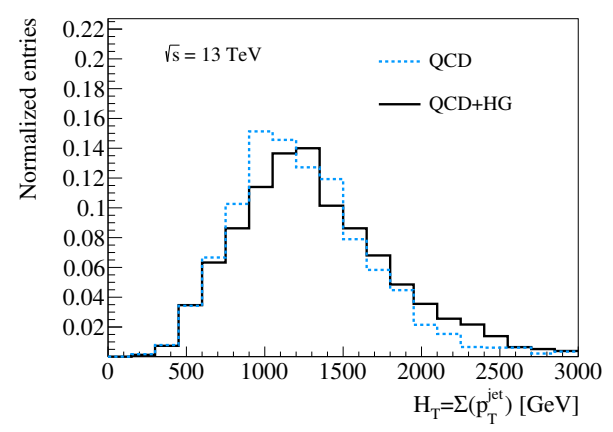

(d)

Figure 5. Kinematical distributions after detector simulation for $\sqrt{s}=13 \mathrm{TeV}$ with $M_{G}=3.5 \mathrm{TeV}$ and $M_{X_{2 / 3}^{\prime}}=1 \mathrm{TeV}$. The distributions shown are the invariant mass of the objects reconstructing $X_{2 / 3}^{\prime}$ (top left), the $Z$ boson transverse momentum (top right), the reconstructed $X_{2 / 3}^{\prime}$ transverse momentum (bottom left) and $H_{\mathrm{T}}$ (bottom right). All distributions are normalized to unit area.

final state objects; the transverse momentum of the $Z$ bosons (top right); the transverse momentum of the reconstructed VLQ (bottom left) and $H_{\mathrm{T}}$ (bottom right). In these plots we have fixed $M_{G}=3.5 \mathrm{TeV}, M_{X_{2 / 3}^{\prime}}=1 \mathrm{TeV}$ and $\sqrt{s}=13 \mathrm{TeV}$ and we show only distributions normalized to unit area. The distributions at $8 \mathrm{TeV}$ are similar to the ones we show, if anything with smaller differences between the QCD and QCD+HG productions. Thus, we see that the somewhat small differences seen at the parton level are almost completely washed out once detector simulation is included. This conclusion remains valid for all the kinematical distributions that we have studied. In the case that the heavy gluon is slightly below the kinematical threshold for pair production and dominates over the QCD production, in which we saw noticeable shape differences between the different production mechanism we have checked that these differences are still visible at the detector level. These kinematical differences are however overwhelmed by the large enhancement in the cross section and have little impact on the final limits.

\subsection{Recasting VLQ searches}

As we have seen in the previous section, the small kinematical differences observed at the parton level between the QCD and QCD+HG production mechanisms are almost completely washed out once detector simulation is included. This means that the efficiencies of 


\begin{tabular}{|l|c|c|}
\hline Selection & QCD cut efficiency (\%) & QCD+HG cut efficiency (\%) \\
\hline Leptonic $Z$ & 1.28 & 1.36 \\
\hline$\geq 2$ jets & 99.82 & 99.90 \\
\hline$\geq 2 b$-jets & 64.25 & 64.00 \\
\hline$p_{\mathrm{T}}(Z)>150 \mathrm{GeV}$ & 93.09 & 92.50 \\
\hline$H_{\mathrm{T}}>600 \mathrm{GeV}$ & 94.19 & 93.42 \\
\hline
\end{tabular}

Table 1. Event selection and cut efficiencies for both QCD and QCD+HG productions at $\sqrt{s}=$ $8 \mathrm{TeV}$ for $M_{G}=3.5 \mathrm{TeV}$ and $M_{X_{2 / 3}^{\prime}}=1 \mathrm{TeV}$. Each efficiency is derived based on the number of events which passed the cut before. The efficiency of the first cut includes the leptonic BR of the $Z$.

the experimental analyses, and therefore the experimental sensitivity, are likely to be very similar in models with and without a heavy gluon in the spectrum. In this section we are going to perform a detailed quantitative analysis of the impact that the small remaining differences have on the final experimental limits. We will do so by computing the expected limits in the $M_{G}-M_{X_{2 / 3}^{\prime}}$ plane in two different ways. The first way is to compute the limits by performing the corresponding analyses on the actual model with both $X_{2 / 3}^{\prime}$ and $G$. The second way is to compute the experimental sensitivity of the analyses assuming only QCD production, as currently done by the LHC experimental collaborations. In this second approach the experimental limit on the corresponding cross section is a function of $M_{X_{2 / 3}^{\prime}}$ only. We will then overlay the theoretical cross section in our model for different values of $M_{G}$, assuming that the efficiencies are identical in both cases and obtaining in this way the limits as a function of $M_{G}$. The first approach corresponds to the properly computed limits, specific for the model at hand. The second approach, on the other hand, relies on the (reasonable, as we have seen) assumption that the experimental efficiencies are quite insensitive to the presence of the HG but has the advantage that it uses the experimental information that LHC collaborations are currently publishing and simply rescaling the theoretical production cross section.

In order to fully exploit the small kinematical differences observed after detector simulation we will apply the procedure described above to two different experimental analyses. The first one is a recast of a search for pair production of VLQ with at least one of them decaying into a $Z$ published by the ATLAS collaboration [20], in which the VLQ is not fully reconstructed. The second one is a more sophisticated multivariate analysis, to take full advantage of the small kinematical differences, in which the VLQ if fully reconstructed. Let us discuss the two analyses and the corresponding limits in turn.

In our first analysis we have replicated the ATLAS selection for the dileptonic channel which is summarized in table 1, together with the efficiency of each cut at $8 \mathrm{TeV}$ for $M_{G}=3.5 \mathrm{TeV}$ and $M_{X_{2 / 3}^{\prime}}=1 \mathrm{TeV}$. First, a pair of opposite-sign same-flavour leptons $\left(p_{T}(\ell)>25 \mathrm{GeV},|\eta(e)|<2.47\right.$, excluding electrons within $1.37<|\eta|<1.52$, and $|\eta(\mu)|<$ $2.5)$ are required with $\left|m_{\ell \ell}-m_{Z}\right|<10 \mathrm{GeV}$. The events passing the first cut are required to have at least 2 jets $\left(p_{T}(j)>25 \mathrm{GeV},|\eta(j)|<5\right)$, with at least two of them being $b$-tagged. 


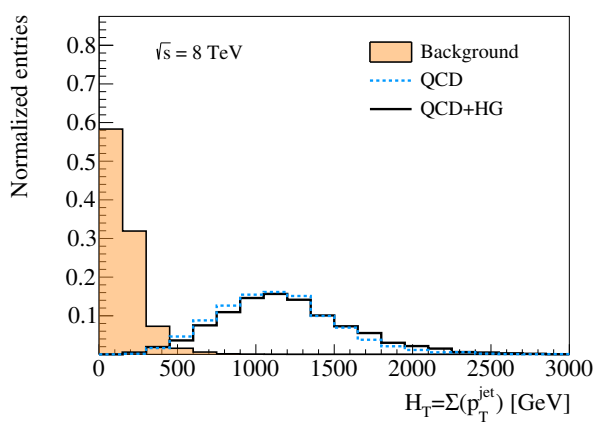

(a)

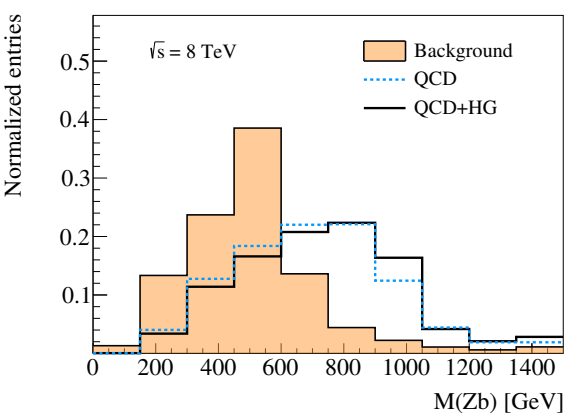

(b)

Figure 6. Signal and background distributions at $\sqrt{s}=8 \mathrm{TeV}$ corresponding to $H_{\mathrm{T}}$ (left) for events passing the first three cuts and and the invariant mass of the $Z b$ system applying all selection (right). The distributions are normalized to unit area. We have fixed $M_{G}=3.5 \mathrm{TeV}$ and $M_{X_{2 / 3}^{\prime}}=1 \mathrm{TeV}$.

Two further cuts are applied to reduce background contamination: $p_{\mathrm{T}}(Z)>150 \mathrm{GeV}$ and $H_{\mathrm{T}}>600 \mathrm{GeV}$, where $H_{\mathrm{T}}$ is the scalar sum of the $p_{\mathrm{T}}$ of all selected jets. As we can see in the table the efficiencies are very similar for the QCD and QCD+HG productions. We have also simulated the two main backgrounds identified by the ATLAS analysis, namely $Z+$ jets and $t \bar{t}$, using the same tools as for the signal. For the $Z+$ jets background we have generated a matched sample of $Z+\mathrm{bb}$ and $Z+\mathrm{cc}$ with up to 3 additional jets in the matrix element. No $Z+$ light sample has been generated since after the $2 b$-jet selection the contribution is negligible. The $t \bar{t}$ sample has been generated at NLO in MG5 and the dileptonic decay has been performed with Madspin [27].

As expected the relevant kinematical distributions after cuts present minimal differences between the QCD and QCD+HG production mechanisms. As an example we show in figure 6 the $H_{\mathrm{T}}$ distribution after the three first cuts (left) and the invariant mass of the $Z b$ system (right), which is the discriminant variable defined as in the ATLAS publication: the $Z$ boson is the candidate selected before and the $b$-jet is the highest- $p_{\mathrm{T}} b$-tagged jet in each event. We also show the corresponding background distibutions for comparison. All distributions are normalized to unit area and we have fixed $M_{G}=3.5 \mathrm{TeV}$ and $M_{X_{2 / 3}^{\prime}}=1 \mathrm{TeV}$. Finally, we show the corresponding limits in the $M_{G}-M_{X_{2 / 3}^{\prime}}$ plane computed with the two methods described above in the left panel of figure 7 . The solid purple line corresponds to the bounds computed within the model with the HG included. The green line, labeled "Scaled QCD limit", corresponds to the bounds computed assuming that the experimental sensitivity is the same in the QCD and QCD+HG production mechanisms. This second method is illustrated in the right panel of figure 7 , in which we show in solid black the experimental limit on the $X_{2 / 3}^{\prime} \bar{X}_{2 / 3}^{\prime}$ production cross section as a function of $M_{X_{2 / 3}^{\prime}}$ assuming QCD production, as well as the theoretical pair production cross section, assuming QCD $+\mathrm{HQ}$ production, for different values of $M_{G}$. The points in which these curves cross the experimental limit determine the solid green curve on the left panel. As we can see in the figure, the two ways of computing the limits give virtually identical results. This indicates that, at least with current analyses at $8 \mathrm{TeV}$ that do not fully reconstruct the VLQ, one can 


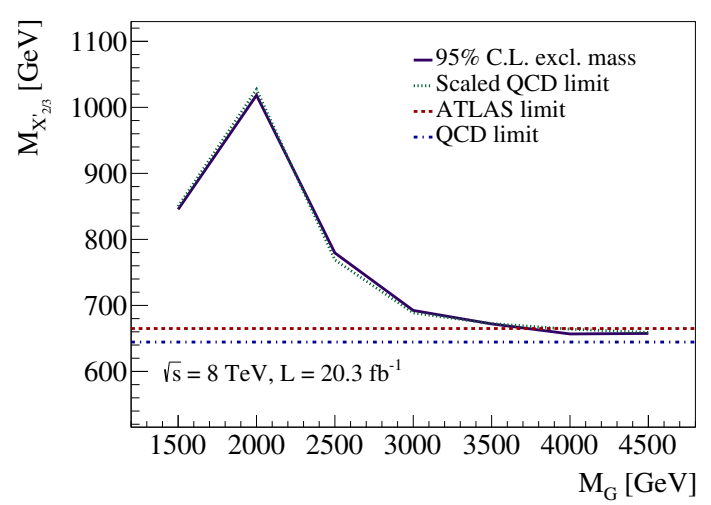

(a)

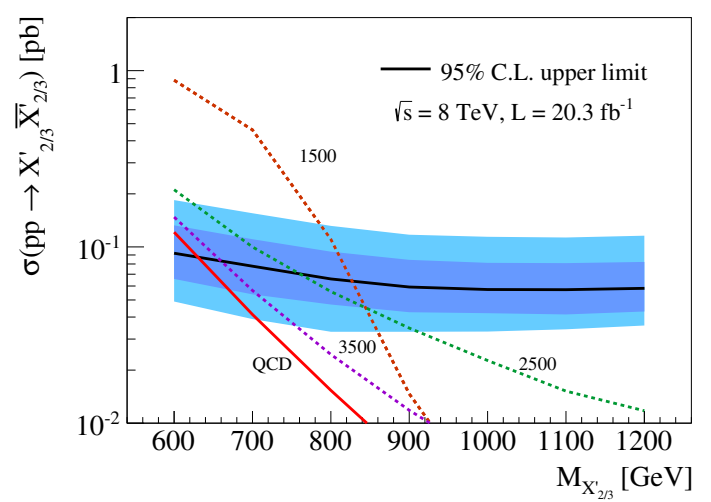

(b)

Figure 7. Left: $95 \%$ confidence level (C.L.) lower limits in the $M_{G}-M_{X_{2 / 3}^{\prime}}$ plane computed with the two methods discussed in the text. We show for comparison the limit reported by ATLAS $(\sqrt{s}=8 \mathrm{TeV})$ in dashed red and the corresponding number that we have obtained with our recast of that analysis in dashed blue. Right: 95\% C.L. upper limits on the pair production cross section as a function of $M_{X_{2 / 3}^{\prime}}$ assuming QCD production (solid black) with 1- and 2- $\sigma$ bands in blue, together with the theoretical production cross sections in the model for different values of $M_{G}$.

simply use the experimental limits on the production cross section as a function of the VLQ mass published by the experimental collaborations to put limits on models with a heavy gluon. We also show in the left panel with horizontal lines the limit we obtain in the case of QCD production and the limit reported for that case by ATLAS. The level of agreement between the two and of them with the large $M_{G}$ limit shows the consistency of our results.

Let us now consider the second, more sophisticated, type of analysis to try to exploit the small kinematical differences observed at the detector level. In this analysis we modify slightly the event selection and reconstruct the $X_{2 / 3}^{\prime}$ decay to a $Z$ boson as follows. The $Z$ boson is reconstructed as in the previous analysis. We then require at least 4 jets with at least 2 of them being $b$-tagged jets. Two of the selected jets are required to have an invariant mass inside a $10 \mathrm{GeV}$ window of $W$ mass and the two leptons reconstructing the $Z$ boson are required to satisfy $\Delta R\left(\ell_{1}, \ell_{2}\right)<1.5$. These two last cuts reduce the background contamination while still leaving the $p_{\mathrm{T}}$ and $H_{\mathrm{T}}$ variables with discriminant power. For the reconstruction of the $W$, top and $X_{2 / 3}^{\prime}$ we use a $\chi^{2}$ to find the best combination of objects reconstructing the desired particles. Specifically, for a particle $X$ which decays into two particles $i, j$, we define $\bar{\chi}_{X}^{2}$ as

$$
\bar{\chi}_{X}^{2}=\frac{\left(M_{i j}-M_{X}\right)^{2}}{\sigma_{X}^{2}} \Delta R(i, j),
$$

where $M_{i j}$ is the invariant mass of the $i, j$ system, $M_{X}$ is the mass of the mother particle, $\sigma_{X}$ is the width of the mother particle and $\Delta R(i, j)$ is the angular separation between both decay products. Using this definition we select the jets and $b$-jet pairing that minimizes

$$
\left(\bar{\chi}_{W}^{2}+\bar{\chi}_{t}^{2}\right) \Delta R(Z, t)
$$




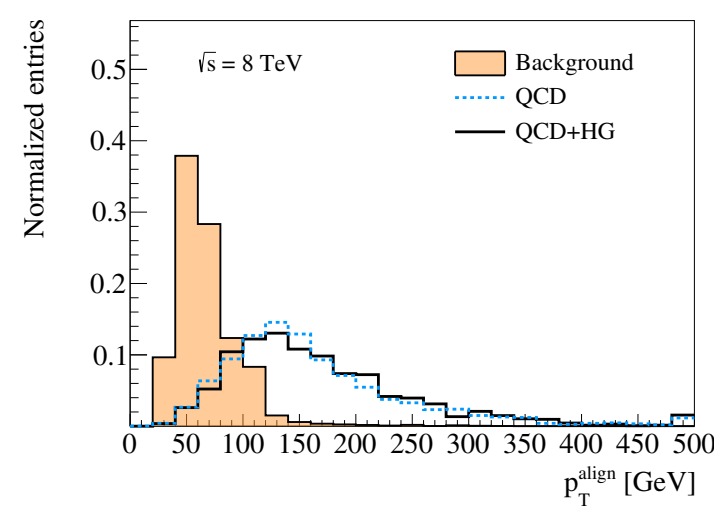

(a)

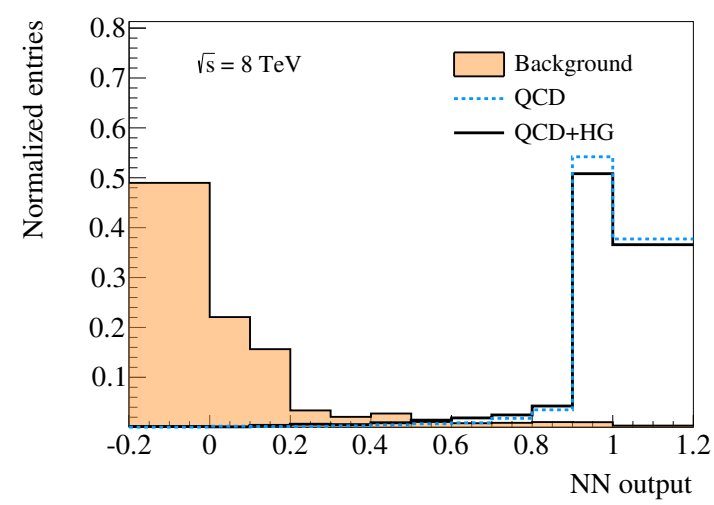

(b)

Figure 8. $p_{\mathrm{T}}^{\text {align }}$ (left) and NN output (right) distributions for signal and background normalized to unit area for $\sqrt{s}=8 \mathrm{TeV}, M_{G}=3.5 \mathrm{TeV}$ and $M_{X_{2 / 3}^{\prime}}=1 \mathrm{TeV}$.

Only hadronic decays of the $W$ boson are considered and the top quark is reconstructed with the reconstructed $W$ boson and a $b$-jet. The $\Delta R$ term in the $\bar{\chi}_{X}^{2}$ definition is used to include the angular separation between the decay products in the object reconstruction.

After this selection is applied we define a multivariate analysis using a neural network $(\mathrm{NN})$ implemented through TMVA [28] with 7 input distributions: jet multiplicity, $p_{\mathrm{T}}(t)$, $p_{\mathrm{T}}\left(X_{2 / 3}^{\prime}\right), p_{\mathrm{T}}(Z), H_{\mathrm{T}}, M_{X_{2 / 3}^{\prime}}$ and $p_{\mathrm{T}}^{\text {align }}$. $p_{\mathrm{T}}^{\text {align }}$ is a variable that is sensitive to both the energy of the decay products of the vector-like quark and the angular separation between them. It increases as we move to a more boosted regime and is defined as

$$
p_{\mathrm{T}}^{\text {align }}=\frac{\sqrt{p_{\mathrm{T}}(Z)^{2}+p_{\mathrm{T}}(W)^{2}+p_{\mathrm{T}}(b)^{2}}}{\max \left(\Delta R\left(X_{2 / 3}^{\prime}, Z\right), \Delta R\left(X_{2 / 3}^{\prime}, W\right), \Delta R\left(X_{2 / 3}^{\prime}, b\right)\right)} .
$$

These variables are selected to provide good discrimination between signal and background and for their potential to provide discrimination between both production mechanisms (QCD and QCD+HG). The NN is trained for each pair of masses of $G$ and $X_{2 / 3}^{\prime}$ quark separately and the output of the $\mathrm{NN}$ is used as discriminant variable to derive exclusion limits. We show in figure 8 the signal and background distributions of $p_{\mathrm{T}}^{\text {align }}$ (left) and the NN output (right) for $\sqrt{s}=8 \mathrm{TeV}, M_{G}=3.5 \mathrm{TeV}$ and $M_{X_{2 / 3}^{\prime}}=1 \mathrm{TeV}$. In both cases the distributions are normalized to unit area.

We use the $C L_{s}$ method [29] with the NN output as the discriminating variable to compute the limits in the $M_{G}-M_{X_{2 / 3}^{\prime}}$ plane with the two methods described at the beginning of this section. The result is shown in figure 9 using the same color coding as in the left panel of figure 7 . We see from the results in the figure that, although the multivariate analysis seems to be more sensitive to the presence of the heavy gluon, the difference between the properly computed limit, taking into account the presence of $\mathrm{HG}$, and the one in which the QCD experimental limit is used is only marginal. The improvement in the actual limits is also minimal. Thus, the conclusion drawn from the previous analysis that one can simply take the experimentally published limits that assume QCD production and 


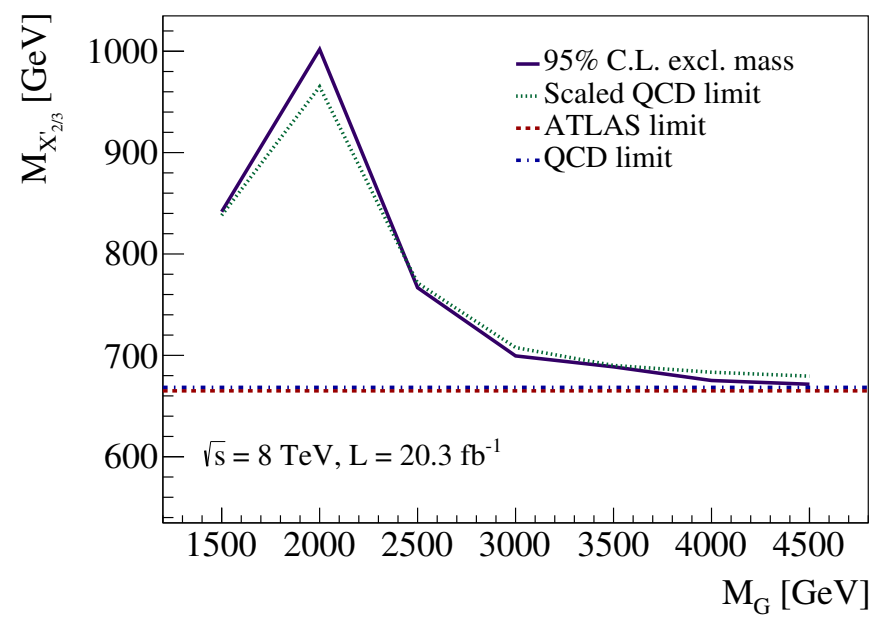

Figure 9. $95 \%$ C.L. lower limit on $M_{X_{2 / 3}^{\prime}}$ as a function of $M_{G}$ for $\sqrt{s}=8 \mathrm{TeV}$ with the full run-1 integrated luminosity. The green line shows the lower mass limit assuming that the only effect is an increase in the cross section and the purple line shows the lower mass limit when considering the correct cross section upper limit for each pair of masses.

use them to compute the bounds on models with a heavy gluon seems to be robust and apply also to more sophisticated analyses with a richer object reconstruction.

\section{Limits on heavy gluons from vector-like quark searches}

Once we have checked in detail that the efficiencies and therefore the experimental limits on the cross section of pair produced VLQ are insensitive to the presence of the heavy gluon we proceed to compute the limits in the $M_{G}-M_{Q}$ plane for our CHM, where now $Q$ stands for any of the VLQ present in the spectrum. In section 4.1 we use the published run-1 limits on VLQ pair production (QCD only) to compute the current bounds on the model assuming the experimental efficiencies to be insensitive to the presence of the heavy gluon. We then proceed in section 4.2 to estimate the bounds that can be obtained from the multivariate analysis described in the previous section with the early run- 2 data (we assume an integrated luminosity of $10 \mathrm{fb}^{-1}$ ).

\subsection{Current limits}

In this section we reinterpret the current results published by the ATLAS and CMS collaborations assuming that the experimental efficiencies are insensitive to the presence of the heavy gluon. We have tried to be as complete as possible using different analyses that target all the VLQ present in the model under study.

When trying to use the published limits we find a first obstacle. We have checked in the previous sections the independence of the efficiencies to the presence of the heavy gluon, however they can be quite sensitive to the decay BR assumed for the VLQ. The reason is cross-contamination between different decay channels. A specific analysis is more sensitive to certain decay channels than others and therefore if the BR change we change 
the population of samples with different efficiencies and thus the global efficiency of the analysis. We have used a specific analysis whenever the BR assumed for the channel that that particular analysis is most sensitive to is equal or smaller than the one in our model. This gives us a conservative bound since we would be assuming in this way a global efficiency that is equal or smaller than the one in our model, due to the contamination of channels with smaller efficiencies. The experimental collaborations have made a tremendous effort in obtaining lower limits on the mass of the VLQ for arbitrary BR. In doing that, they have computed the corresponding limits in the production cross section as a function of the VLQ mass. It would be extremelly useful to provide that information too, which would allow us to compute the limits in models with heavy gluons using the correct BR.

A second difficulty arises from the fact that the experimental limits are always reported assuming that only one VLQ is present in the spectrum. In our case, due to the specific BR pattern, we can group the VLQ in two different groups, $X_{5 / 3}$ and $B$ both decay into $W t$ and, unless full reconstruction of one decay leg with same/opposite sign leptons is performed, they are difficult to distinguish (see [30] for studies on how to characterize the different top partners in a similar model, assuming QCD production). A second group is formed by $X_{2 / 3}^{\prime}$ and $T^{\prime}$, which have the exact same decay patterns. In our model, $X_{5 / 3}$ and $X_{2 / 3}^{\prime}$ are degenerate and lighter than $T^{\prime}$ and $B$. In the region of parameter space that we are exploring the mass splitting is of the order of $10 \%$, which is large enough to make interference effects small but not so large that two distinct peaks would show up in the relevant distributions. Furthermore, the heavier ones, $T^{\prime}$ and $B$, are heavy enough to make their contribution to the total cross section much smaller than the one from the lighter ones, $X_{5 / 3}$ and $X_{2 / 3}^{\prime}$, in the searches to which both contribute. We show in the left panel of figure 10 the $T^{\prime}$ mass as a function of the $X_{2 / 3}^{\prime}$ mass, for the chosen parameters. Thus, the global efficiencies are likely to be quite insensitive to their presence. In order to be quantitative, we have repeated our multivariate analysis including the two charge $2 / 3$ quarks, $X_{2 / 3}^{\prime}$ and $T^{\prime}$, for a few selected points. We show in the right panel of figure 10 a comparison between the experimental sensitivity (limit on the production cross section) obtained in the full model with both quarks and the one obtained neglecting the presence of $T^{\prime}$. As we can see the two curves are compatible within uncertainties and we can therefore compute the limits overlaying the total cross section (including $X_{2 / 3}^{\prime}$ and $T^{\prime}$ ) over the published experimental bounds on the production cross section.

We have used the following experimental results to compute the current limits on the VLQ present in out model. For $B$ we have used the results published in [31] (ATLAS) and [32] (CMS). The ATLAS analysis considers a singlet model with different BR than in our case. However, it is very sensitive to the $W$ decay and, as discussed, we have conservatively rescaled their result to our $100 \% \mathrm{BR}$ into $W t$. The CMS result is already published for a BR of $100 \%$ to the $W$ channel. Unfortunately, the mass of the $B$ quark in our model is, for the range of parameters we have considered, typically heavier than the masses these analyses are sensitive to and we cannot therefore report bounds for all the $M_{G}$ values. We can nevertheless provide the corresponding limits in some cases. For instance, using the ATLAS results we can exclude values of $M_{B}$ ranging from $M_{B} \simeq 750 \mathrm{GeV}$ for $M_{G}=4 \mathrm{TeV}$ to $M_{B} \simeq 1 \mathrm{TeV}$ for $M_{G}=2 \mathrm{TeV}$. Using the CMS results we can excluded values of $M_{B}$ ranging 


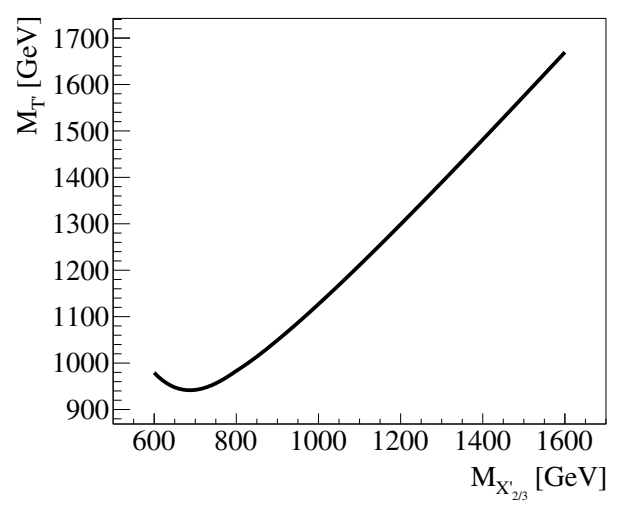

(a)

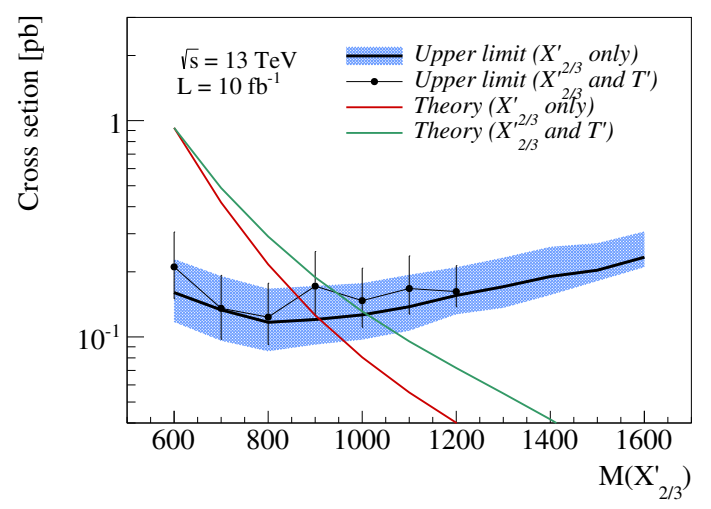

(b)

Figure 10. Left: $M_{T^{\prime}}$ mass as a function of $M_{X_{2 / 3}^{\prime}}$ mass for the chosen parameters. Right: comparison between the $95 \%$ C.L. experimental limit obtained assuming only $X_{2 / 3}^{\prime}$ production (black solid line) and assuming production of both $X_{2 / 3}^{\prime}$ and $T^{\prime}$ (black dots). The solid red line represents the cross section of pair production of $X_{2 / 3}^{\prime}$ only while the dashed green line represent the cross section of pair production of both $X_{2 / 3}^{\prime}$ and $T^{\prime}$. In this figure $M_{G}=3.5 \mathrm{TeV}$ and $\sqrt{s}=13 \mathrm{TeV}$ have been chosen as an illustrative example. Similar behaviour is found for different masses and center-of-mass energy.

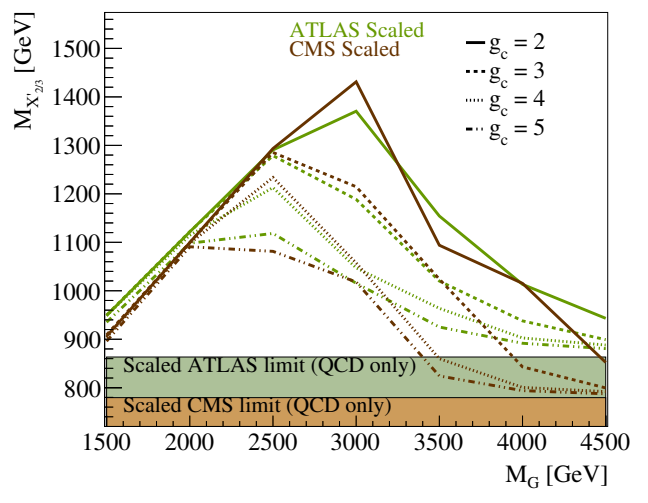

(a)

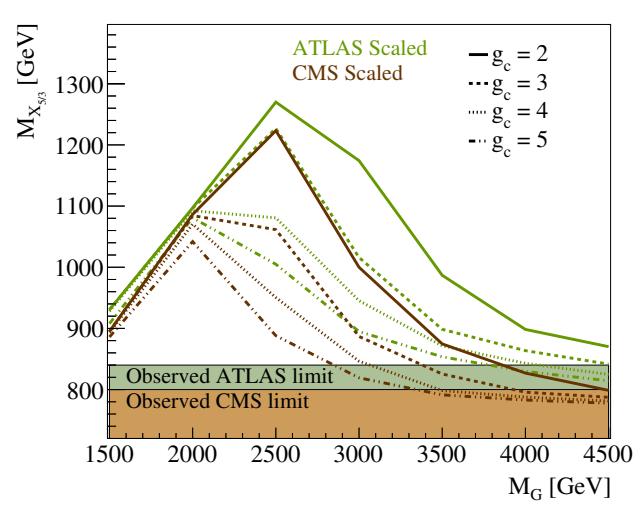

(b)

Figure 11. $95 \%$ C.L. lower limits on the $M_{G}-M_{X_{2 / 3}^{\prime}}$ (left) and $M_{G}-M_{X_{5 / 3}}$ (right) planes, derived from full run-1 published data $(\sqrt{s}=8 \mathrm{TeV})$, for different values of $g_{c}$. The filled orange and green region represent the limits for CMS and ATLAS, respectively, assuming QCD production of $X_{2 / 3}^{\prime}$ and $T^{\prime}$ (left) and $X_{5 / 3}$ (right). 


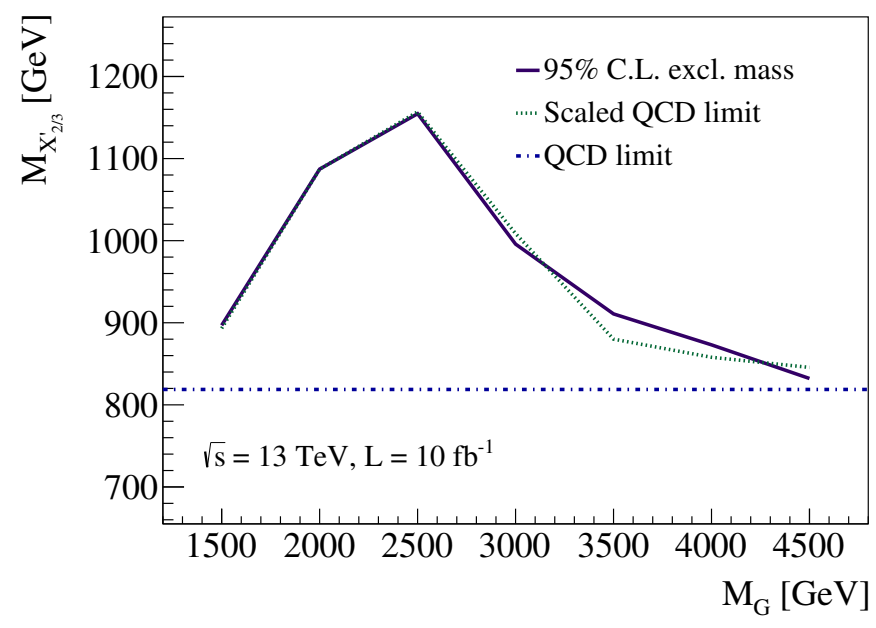

Figure 12. $95 \%$ C.L. lower limit on the $X_{2 / 3}^{\prime}$ mass as a function of $M_{G}$ at $\sqrt{s}=13 \mathrm{TeV}$ and an integrated luminosity of $10 \mathrm{fb}^{-1}$. The green line shows the lower mass limit assuming that the only effect is an increase in the cross section and the purple line shows the lower mass limit when considering the correct cross section upper limit for each pair of masses. The dashed blue line shows the lower mass limit when only QCD production is assumed.

from $M_{B} \simeq 800 \mathrm{GeV}$ for $M_{G}=3 \mathrm{TeV}$ to $M_{B} \simeq 950 \mathrm{TeV}$ for $M_{G}=2 \mathrm{TeV}$. These limits have been derived assuming $g_{c}=3$. For the $X_{2 / 3}^{\prime}$ and $T^{\prime}$ quarks we have used the results in [3] (ATLAS) and [4] (CMS). The ATLAS results contain a combination of two analyses that target decays of the VLQ into $H$ and $W$, respectively. Since charged current decays are not present in our model we use the result quoted for the doublet (same BR as in our model) only in the $H$ channel. The CMS analysis reports limits for a 2:1:1 BR pattern into $W, H$ and $Z$, respectively, and the analysis used is very sensitive to the $Z$ channels. Thus, we conservatively scale their results to out BR as discussed above. The results can be found in the left panel of figure 11 for different values of $g_{c}$. The corresponding value of $M_{T^{\prime}}$ can be obtained from figure 10. In the case of the $X_{5 / 3}$ the same ATLAS analysis used for the $B$ quark published results for the search of a vector-like quark with a electric charge of $5 / 3$ assuming a BR of $100 \%$ to $W t$. We have also used the CMS analysis [33] searching for VLQ with an electric charge of $5 / 3$ in the same-sign dilepton final state. They assume a $100 \%$ BR into $W t$ so we can directly use their results, which can be found in the right panel of figure 11.

\subsection{Early run-2 expectations}

Once we have computed the current bounds in the $M_{G}-M_{Q}$ plane with $Q$ running over all the VLQ present in our model we would like to compare them with the reach of a run-2 early data set. The goal is two-fold, first we would like to see how much the limits can improve with a few $\mathrm{fb}^{-1}$ of integrated luminosity at $\sqrt{s}=13 \mathrm{TeV}$ with respect to the current ones; second we would like to explore the sensitivity to the presence of the heavy gluon at the initial stages of a new run with higher energy, as opposed to the analyses we have performed so far in which the limits are at the verge of the kinematical reach. 
We take as a benchmark analysis the multivariate one we presented in section 3.2 and estimate its reach in the $M_{G}-M_{X_{2 / 3}^{\prime}}$ plane at $\sqrt{s}=13 \mathrm{TeV}$ and an integrated luminosity of $10 \mathrm{fb}^{-1}$. We use the same selection and tools and compute the limits again in two ways, either including QCD+HG from the start or computing the upper limits in the cross section assuming QCD production and rescaling the corresponding cross section. Our results are summarized in figure 12, in which we show the $95 \%$ C.L. lower bounds in mass for the $X_{2 / 3}^{\prime}$ quark as a function of the $G$ mass. Two points are worth emphasizing. The first point is that the limits computed by a simple rescaling of the cross section (using the results that assume QCD production as would be published by the experimental collaborations) are perfectly compatible with the full ones computed with the model at hand. Thus, even when exploring regions of parameter space far from the kinematical reach of the experiment, it seems like the experimental limits on the pair production cross section of VLQ is quite insensitive to the presence of the heavy gluon. It is therefore perfectly legitimate to use them to put bounds on any model in which heavy gluons contribute to the VLQ production. A second important point is that there is, already with $10 \mathrm{fb}^{-1}$ of integrated luminosity, a significant improvement in the limits with respect to current ones. The early run-2 limits on the mass of the VLQ are in the $\sim 820-1160 \mathrm{GeV}$ range (to be compared with the equivalent $\sim 660-1000 \mathrm{GeV}$ at $\sqrt{s}=8 \mathrm{TeV})$. A similar improvement can be expected with respect to the complete current limits presented in figure 11. Given these two points, it is clear that as soon as the experimental collaborations publish their results on VLQ production during run-2, they can be easily translated to bounds on the parameter space of models with heavy vectors in the spectrum.

\section{Conclusions}

We have considered in this article the problem of reinterpretation of experimental results in terms of models different from the ones used by the experimental collaborations. Such reinterpretations are always delicate since it is difficult to assess the impact of the new features of the model in the final results. Assuming that the signatures of the two models are reasonably similar, there are, broadly speaking, two options. The first option is that the distribution for the kinematical observables used in the analysis are very similar in both models. In that case the experimental sensitivity (limit on the production cross section as a function of the relevant scale) will be very similar for both models and the limits can be computed by a simple rescaling of the theoretical cross section. The alternative option is that some observables show significant differences between the two models. The reinterpretation of the limits in the new model become then much more complicated, and the only possibility is to recast the corresponding analysis using fast detector simulation tools. This latter case is not completely satisfactory, as it does not take full advantage of the experimental publication and requires a certain degree of mastery in the implementation of the model and the use of Monte Carlo and detector simulation tools. On the other hand, it offers extra handles on how to more efficiently search for the new model.

For the sake of concreteness we have considered pair production of new VLQ in CHM. A common occurence in these models is the presence of a heavy color octet vector boson, heavy gluon, that can contribute to the pair production of the VLQ. We have performed 
a detailed quantitative study of the effect that the heavy gluon has on the distributions of different kinematical variables, both at parton level and after detector simulation. The small differences found at the parton level in variables that are relatively up in the decay chain are almost completely washed out after detector simulation. Thus, in practice, the limits on the VLQ and heavy gluon masses that one obtains by using the full model and the ones that use just QCD production (neglecting the heavy gluon) to compute the limit on the production cross section are virtually identical. The latter has the precious advantage that it uses directly the published limits computed by the experimental collaborations and require only a minimal amount of Monte Carlo simulations to compute the corresponding theoretical cross sections at the parton level.

We have tested the validity of this approximation in two different regimes of an experiment, one in which the limits are close to the kinematical reach of the experiment and another in which a new energy frontier is being explored for the first time. These correspond to the full LHC run-1 data and the early run-2 data, respectively. In both cases we have found that the experimental limits on the production cross section are quite insensitive to the presence of the heavy gluon and therefore the experimental results can easily be reinterpreted in models with new VLQ and heavy gluons. This extends not only to current analyses but also to more sophisticated analyses in which full kinematical information, including the reconstruction of the VLQ, is used. Presumably only a full reconstruction of the VLQ pair invariant mass could give a significant sensitivity to the presence of the heavy gluon.

We have reached these conclusions by performing an exhaustive set of comparisons of kinematical distributions and detailed statistical analyses, which include multivariate techniques. We have found that the main degradation of the HG signal occurs at the reconstruction level, due in part to a wrong assignment of physical objects by the reconstruction algorithms in the presence of boosted topologies due to the overlap of the considered jets. The use of boosted techniques might partially compensate this effect but a detailed analysis, which is beyond the scope of this study, would be needed to assess in a quantitative way the discriminating power of a reconstruction method using larger jets and jet substructure variables. In any case, our modeling of the detector is based on fast detector simulation and therefore our conclusions are limited by the inherent accuracy of such a tool. In that sense it would be very important that experimental collaborations consider the possibility of comparing the experimental bounds on the VLQ pair production cross section with and without a heavy gluon.

We would also like to encourage the LHC experimental collaborations to report their limits on the VLQ pair-production cross section as a function of the VLQ BR. They are available to the collaborations, as they are used to compute the limits on the VLQ masses for arbitrary BR. However they are not publicly available, thus limiting the reinterpretation of their limits to other models, e.g. with or without heavy gluons.

\section{Acknowledgments}

We would like to thank G. Perez for useful discussions and motivation at the initial stages of this work. JS is supported by MINECO, under grant numbers FPA2010-17915 and FPA2013-47836-C3-2-P, by the European Commission through the contract PITN-GA- 
2012-316704 (HIGGSTOOLS) and by Junta de Andalucía grants FQM 101 and FQM 6552. JS thanks the Pauli Center Visitor Program for financial support. JPA and NFC are supported by FEDER, COMPETE-QREN and FCT, Portugal, through grant SFRH/BD/52002/2012 (JPA) and contract IF/00050/2013 (NFC).

\section{A Technical details of the model}

In this appendix we detail the different terms appearing in the Lagrangian eq. (2.5). The covariant derivatives read explicitly

$$
\begin{aligned}
\mathrm{i} D_{\mu} q_{L} & =\left(\mathrm{i} \partial_{\mu}+g \frac{\sigma^{i}}{2} W_{\mu}^{i}+\frac{g^{\prime}}{6} B_{\mu}+g_{e} G_{\mu}^{e}\right) q_{L} \\
\mathrm{i} D_{\mu} t_{R} & =\left(\mathrm{i} \partial_{\mu}+\frac{2 g^{\prime}}{3} B_{\mu}+g_{c} G_{\mu}^{c}\right) t_{R} \\
\mathrm{i} D_{\mu} \Psi & =\left(\mathrm{i} \partial_{\mu}+\frac{2 g^{\prime}}{3} B_{\mu}+g_{c} G_{\mu}^{c}\right) \Psi .
\end{aligned}
$$

The remaining terms read

$$
\begin{aligned}
\mathrm{i} \bar{\Psi}_{R}^{i} d_{i} t_{R}= & \frac{g}{\sqrt{2}} s_{h}\left[\left(\bar{X}_{5 / 3}\right)_{R} W^{+}-\bar{B}_{R} W^{-}\right] t_{R} \\
& -\frac{g}{2 c_{W}} s_{h}\left[\bar{T}_{R}+\left(\bar{X}_{2 / 3}\right)_{R}\right] Z t_{R}+\mathrm{i}\left[\left(\bar{X}_{2 / 3}\right)_{R}-\bar{T}_{R}\right] \frac{\not \rho \rho}{f} t_{R}, \\
\bar{\Psi}\left(\frac{2 g^{\prime}}{3} \not B-\phi\right) \Psi= & \frac{g}{c_{W}}\left(-\frac{1}{2}+\frac{s_{W}^{2}}{3}\right) \bar{B} \not Z B+\frac{g}{c_{W}}\left(\frac{1}{2}-\frac{5 s_{W}^{2}}{3}\right) \bar{X}_{5 / 3} \not X_{5 / 3} \\
& +\frac{g}{c_{W}}\left(\frac{1}{2} c_{h}-\frac{2 s_{W}^{2}}{3}\right) \bar{T} \not Z T+\frac{g}{c_{W}}\left(-\frac{1}{2} c_{h}-\frac{2 s_{W}^{2}}{3}\right) \bar{X}_{2 / 3} Z X_{2 / 3} \\
& +\frac{g}{\sqrt{2}}\left\{\bar{B} W^{-}\left[c_{h / 2}^{2} T+s_{h / 2}^{2} X_{2 / 3}\right]+\bar{X}_{5 / 3} W^{+}\left[s_{h / 2}^{2} T+c_{h / 2}^{2} X_{2 / 3}\right]+\text { h.c. }\right\} \\
& +\operatorname{photon} \operatorname{couplings}, \\
\left(\bar{Q}_{L}^{5}\right)^{I} U_{I i} \Psi_{R}^{i}= & \bar{b}_{L} B_{R}+\bar{t}_{L}\left[c_{h / 2}^{2} T_{R}+s_{h / 2}^{2}\left(X_{2 / 3}\right)_{R}\right] \\
\left(\bar{Q}_{L}^{5}\right)^{I} U_{I 5} t_{R}= & -\frac{1}{\sqrt{2}} s_{h} \bar{t}_{L} t_{R},
\end{aligned}
$$

where we have denoted

$$
s_{x} \equiv \sin \frac{x}{f}, \quad c_{x} \equiv \cos \frac{x}{f},
$$

except for $s_{W}$ and $c_{W}$, which are the sine and cosine of the Weinberg angle. $\rho$ is the physical Higgs boson and $h$ reads, in the unitary gauge

$$
h \equiv\langle h\rangle+\rho,
$$

with

$$
f s_{\langle h\rangle}=v \approx 246 \mathrm{GeV} .
$$

These terms fix the electroweak couplings of the top and its partners and also the top mass and mixing. In particular the latter two have some implications on the degree of compositeness of the top quark and therefore on its couplings to the heavy gluon. 
Open Access. This article is distributed under the terms of the Creative Commons Attribution License (CC-BY 4.0), which permits any use, distribution and reproduction in any medium, provided the original author(s) and source are credited.

\section{References}

[1] ATLAS collaboration, Observation of a new particle in the search for the Standard Model Higgs boson with the ATLAS detector at the LHC, Phys. Lett. B 716 (2012) 1 [arXiv: 1207.7214] [INSPIRE].

[2] CMS collaboration, Observation of a new boson at a mass of $125 \mathrm{GeV}$ with the CMS experiment at the LHC, Phys. Lett. B 716 (2012) 30 [arXiv:1207.7235] [INSPIRE].

[3] ATLAS collaboration, Search for production of vector-like quark pairs and of four top quarks in the lepton-plus-jets final state in pp collisions at $\sqrt{s}=8 \mathrm{TeV}$ with the ATLAS detector, JHEP 08 (2015) 105 [arXiv: 1505.04306] [INSPIRE].

[4] CMS collaboration, Inclusive search for a vector-like $T$ quark with charge $2 / 3$ in $p p$ collisions at $\sqrt{s}=8 \mathrm{TeV}$, Phys. Lett. B 729 (2014) 149 [arXiv:1311.7667] [inSPIRE].

[5] LHC New Physics Working Group collaboration, D. Alves, Simplified models for LHC new physics searches, J. Phys. G 39 (2012) 105005 [arXiv:1105.2838] [INSPIRE].

[6] D.B. Kaplan and H. Georgi, $\mathrm{SU}(2) \times \mathrm{U}(1)$ breaking by vacuum misalignment, Phys. Lett. B 136 (1984) 183 [INSPIRE].

[7] D.B. Kaplan, Flavor at SSC energies: a new mechanism for dynamically generated fermion masses, Nucl. Phys. B 365 (1991) 259 [INSPIRE].

[8] O. Matsedonskyi, G. Panico and A. Wulzer, Light top partners for a light composite Higgs, JHEP 01 (2013) 164 [arXiv:1204.6333] [INSPIRE].

[9] K. Agashe, A. Delgado, M.J. May and R. Sundrum, RS1, custodial isospin and precision tests, JHEP 08 (2003) 050 [hep-ph/0308036] [INSPIRE].

[10] M. Chala, J. Juknevich, G. Perez and J. Santiago, The elusive gluon, JHEP 01 (2015) 092 [arXiv: 1411.1771] [INSPIRE].

[11] R. Barcelo, A. Carmona, M. Chala, M. Masip and J. Santiago, Single vectorlike quark production at the LHC, Nucl. Phys. B 857 (2012) 172 [arXiv:1110.5914] [INSPIRE].

[12] C. Bini, R. Contino and N. Vignaroli, Heavy-light decay topologies as a new strategy to discover a heavy gluon, JHEP 01 (2012) 157 [arXiv:1110.6058] [INSPIRE].

[13] A. Azatov, D. Chowdhury, D. Ghosh and T.S. Ray, Same sign di-lepton candles of the composite gluons, JHEP 08 (2015) 140 [arXiv: 1505.01506] [INSPIRE].

[14] N. Vignaroli, Z-peaked excess from heavy gluon decays to vectorlike quarks, Phys. Rev. D 91 (2015) 115009 [arXiv:1504.01768] [INSPIRE].

[15] K. Agashe, R. Contino and A. Pomarol, The minimal composite Higgs model, Nucl. Phys. B 719 (2005) 165 [hep-ph/0412089] [INSPIRE].

[16] A. De Simone, O. Matsedonskyi, R. Rattazzi and A. Wulzer, A first top partner hunter's guide, JHEP 04 (2013) 004 [arXiv: 1211.5663] [INSPIRE].

[17] R. Barcelo, A. Carmona, M. Masip and J. Santiago, Stealth gluons at hadron colliders, Phys. Lett. B 707 (2012) 88 [arXiv:1106.4054] [InSPIRE]. 
[18] D. Greco and D. Liu, Hunting composite vector resonances at the LHC: naturalness facing data, JHEP 12 (2014) 126 [arXiv:1410.2883] [INSPIRE].

[19] A. Carmona, A. Delgado, M. Quirós and J. Santiago, Diboson resonant production in non-custodial composite Higgs models, JHEP 09 (2015) 186 [arXiv:1507.01914] [INSPIRE].

[20] ATLAS collaboration, Search for pair and single production of new heavy quarks that decay to a $Z$ boson and a third-generation quark in pp collisions at $\sqrt{s}=8$ TeV with the ATLAS detector, JHEP 11 (2014) 104 [arXiv:1409.5500] [INSPIRE].

[21] C. Degrande, C. Duhr, B. Fuks, D. Grellscheid, O. Mattelaer and T. Reiter, UFO - the Universal FeynRules Output, Comput. Phys. Commun. 183 (2012) 1201 [arXiv:1108.2040] [INSPIRE].

[22] A. Alloul, N.D. Christensen, C. Degrande, C. Duhr and B. Fuks, FeynRules $2.0-a$ complete toolbox for tree-level phenomenology, Comput. Phys. Commun. 185 (2014) 2250 [arXiv: 1310.1921] [INSPIRE].

[23] J. Alwall et al., The automated computation of tree-level and next-to-leading order differential cross sections and their matching to parton shower simulations, JHEP 07 (2014) 079 [arXiv: 1405.0301] [INSPIRE].

[24] T. Sjöstrand, S. Mrenna and P.Z. Skands, PYTHIA 6.4 physics and manual, JHEP 05 (2006) 026 [hep-ph/0603175] [INSPIRE].

[25] DELPHES 3 collaboration, J. de Favereau et al., DELPHES 3, a modular framework for fast simulation of a generic collider experiment, JHEP 02 (2014) 057 [arXiv:1307.6346] [INSPIRE].

[26] M. Cacciari, G.P. Salam and G. Soyez, FastJet user manual, Eur. Phys. J. C 72 (2012) 1896 [arXiv:1111.6097] [INSPIRE].

[27] P. Artoisenet, R. Frederix, O. Mattelaer and R. Rietkerk, Automatic spin-entangled decays of heavy resonances in Monte Carlo simulations, JHEP 03 (2013) 015 [arXiv:1212.3460] [INSPIRE].

[28] A. Hocker et al., TMVA - Toolkit for MultiVariate data Analysis, PoS (ACAT) 040 [physics/0703039] [INSPIRE].

[29] A.L. Read, Presentation of search results: the $C L_{s}$ technique, J. Phys. G 28 (2002) 2693 [INSPIRE].

[30] J.A. Aguilar-Saavedra, Identifying top partners at LHC, JHEP 11 (2009) 030 [arXiv:0907.3155] [INSPIRE].

[31] ATLAS collaboration, Search for vectorlike B quarks in events with one isolated lepton, missing transverse momentum and jets at $\sqrt{s}=8 \mathrm{TeV}$ with the ATLAS detector, Phys. Rev. D 91 (2015) 112011 [arXiv:1503.05425] [INSPIRE].

[32] CMS collaboration, Search for a vector-like bottom quark partner in same sign di-lepton final states, CMS-PAS-B2G-12-020, CERN, Geneva Switzerland (2012).

[33] CMS collaboration, Search for top-quark partners with charge $5 / 3$ in the same-sign dilepton final state, Phys. Rev. Lett. 112 (2014) 171801 [arXiv:1312.2391] [INSPIRE]. 